\title{
Ramanujan Coverings of Graphs *
}

\author{
Chris Hall $^{\dagger}$ \\ Department of Mathematics \\ University of Wyoming, USA \\ chall14@uwyo.edu
}

\author{
Doron Puder \\ School of Mathematics \\ Institute for Advanced Study, \\ USA \\ doronpuder@gmail.com
}

\author{
William F. Sawin ${ }^{\S}$ \\ Department of Mathematics \\ Princeton University, USA \\ wsawin@math.princeton.edu
}

\begin{abstract}
Let $G$ be a finite connected graph, and let $\rho$ be the spectral radius of its universal cover. For example, if $G$ is $k$-regular then $\rho=2 \sqrt{k-1}$. We show that for every $r$, there is an $r$ covering (a.k.a. an $r$-lift) of $G$ where all the new eigenvalues are bounded from above by $\rho$. It follows that a bipartite Ramanujan graph has a Ramanujan $r$-covering for every $r$. This generalizes the $r=2$ case due to Marcus, Spielman and Srivastava (2013).

Every $r$-covering of $G$ corresponds to a labeling of the edges of $G$ by elements of the symmetric group $S_{r}$. We generalize this notion to labeling the edges by elements of various groups and present a broader scenario where Ramanujan coverings are guaranteed to exist.

In particular, this shows the existence of richer families of bipartite Ramanujan graphs than was known before. Inspired by Marcus-Spielman-Srivastava, a crucial component of our proof is the existence of interlacing families of polynomials for complex reflection groups. The core argument of this component is taken from Marcus-Spielman-Srivastava (2015).

Another important ingredient of our proof is a new generalization of the matching polynomial of a graph. We define the $r$-th matching polynomial of $G$ to be the average matching polynomial of all $r$-coverings of $G$. We show this polynomial shares many properties with the original matching polynomial. For example, it is real rooted with all its roots inside $[-\rho, \rho]$.
\end{abstract}

\footnotetext{
${ }^{*}$ A full version of this paper is available at arXiv:1506.02335v2

${ }^{\dagger}$ Partially supported by Simons Foundation award 245619 and IAS NSF grant DMS-1128155

${ }^{\ddagger}$ Supported by the Rothschild fellowship and by the National Science Foundation under agreement No. DMS-1128155

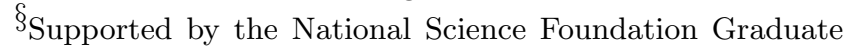
Research Fellowship under Grant No. DGE-1148900
}

Permission to make digital or hard copies of all or part of this work for personal or classroom use is granted without fee provided that copies are not made or distributed for profit or commercial advantage and that copies bear this notice and the full citation on the first page. Copyrights for components of this work owned by others than ACM py otherwise, or republish, to post on servers or to redistribute to lists, requires prior specific permission and/or a fee. Request permissions from Permissions@ acm.org.

STOC'16, June 19-21, 2016, Cambridge, MA, USA

(c) 2016 ACM. $978-1-4503-4132-5 / 16 / 06 \ldots \$ 15.00$

http://dx.doi.org/10.1145/2897518.2897574

\section{Categories and Subject Descriptors}

G.2.2 [Mathematics of Computing]: Discrete Mathematics - Graph Theory; F.2.1 [Analysis of algorithms and problem complexity]: Numerical Algorithms and Problems-Computations on matrices

\section{General Terms}

Theory

\section{Keywords}

Ramanujan graphs, expander graphs, interlacing polynomials, matching polynomial, graph coverings, graph lifts

\section{INTRODUCTION}

Let $G$ be a finite, connected, undirected graph on $n$ vertices and let $A_{G}$ be its adjacency matrix. The eigenvalues of $A_{G}$ are real and we denote them by

$$
\lambda_{n} \leq \ldots \leq \lambda_{2} \leq \lambda_{1}=\mathfrak{p} \mathfrak{f}(G),
$$

where $\lambda_{1}=\mathfrak{p f}(G)$ is the Perron-Frobenius eigenvalue ${ }^{1}$ of $A_{G}$, referred to as the trivial eigenvalue. For example, $\mathfrak{p f}(G)=$ $k$ for $G k$-regular. The smallest eigenvalue, $\lambda_{n}$, is at least $-\mathfrak{p f}(G)$, with equality if and only if $G$ is bipartite. Denote by $\lambda(G)$ the largest absolute value of a non-trivial eigenvalue, namely $\lambda(G)=\max \left(\lambda_{2},-\lambda_{n}\right)$. It is well know that $\lambda(G)$ provides a good estimate to different expansion properties of $G$ : the smaller $\lambda(G)$ is, the better expanding $G$ is (we elaborate in Section 2.1).

However, $\lambda(G)$ cannot be arbitrarily small. Let $\rho(G)$ be the spectral radius of the universal covering tree ${ }^{2}$ of $G$. For instance, if $G$ is $k$-regular then $\rho(G)=2 \sqrt{k-1}$. It is known that $\lambda(G)$ cannot be much smaller then $\rho(G)$, so graphs with $\lambda(G) \leq \rho(G)$ are considered optimal expanders. Following [15, 12] they are called Ramanujan graphs, and the interval $[-\rho(G), \rho(G)]$ called the Ramanujan interval. In the bipartite case, $\lambda(G)=\left|\lambda_{n}\right|=\mathfrak{p f}(G)$ is large, but $G$ can still expand well in many senses (see Section 2.1), and the optimal scenario is when all other eigenvalues are within the Ramanujan interval, namely, when $\lambda_{n-1}, \lambda_{n-2}, \ldots, \lambda_{2} \in[-\rho(G), \rho(G)]$. We call a bipartite graph with this property a bipartite Ramanujan graph.

\footnotetext{
${ }^{1}$ We explain all notions in Section 2.

${ }^{2}$ Roughly speaking, this is the tree of non-backtracking walks in the graph $G$, and its spectral radius is the operator norm of its (infinite) adjacency matrix. See Section 2.1 for more details.
} 


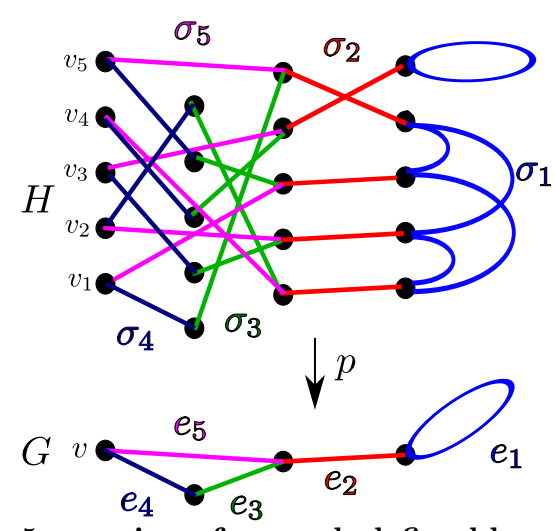

Figure 1: A 5-covering of a graph defined by permutations

Although $G$ is undirected, we regard it as an oriented graph. More precisely, we choose an orientation for each edge in $G$, and we write $E^{+}(G)$ for the resulting set of oriented edges and $E^{-}(G)$ for the edges with the opposite orientation. Finally, if $e$ is an edge in $E^{ \pm}(G)$, then we write $-e$ for the corresponding edge in $E^{\mp}(G)$ with the opposite orientation, and we identify $E(G)$ with the disjoint union $E^{+}(G) \sqcup E^{-}(G)$. We let $h(e)$ and $t(e)$ denote the head vertex and tail vertex of $e \in E(G)$, respectively. We say that $G$ is an oriented undirected graph.

Throughout this paper, the family of r-coverings (at some references called also $r$-lifts) of the graph $G$ is defined via the following natural model, introduced in [1] and [4]. The vertex set of every $r$-covering $H$ is

$$
\left\{v_{i} \mid v \in V(G), 1 \leq i \leq r\right\} .
$$

Its edges are defined via a function $\sigma: E(G) \rightarrow S_{r}$ from the edges to the symmetric group on $[r]$, satisfying $\sigma(-e)=$ $\sigma(e)^{-1}$ (occasionally, we denote $\sigma(e)$ by $\sigma_{e}$ ): for every $e \in$ $E^{+}(G)$ we introduce in $H$ the $r$ edges connecting $h(e)_{i}$ to $t(e)_{\sigma_{e}(i)}$ for $1 \leq i \leq r$. The covering map $p: H \rightarrow G$ is the map defined by $v_{i} \mapsto v$ and $\left(h(e)_{i}, t(e)_{\sigma_{e}(i)}\right) \mapsto e$. See Figure 1.

Definition 1.1. Denote by $\mathcal{C}_{\mathbf{r}, \mathbf{G}}$ the probability space consisting of all r-coverings $\left\{\sigma: E(G) \rightarrow S_{r} \mid \sigma(-e)=\sigma(e)^{-1}\right\}$, endowed with uniform distribution.

Let $H$ be an $r$-covering of $G$ with covering map $p: H \rightarrow G$. If $f: V(G) \rightarrow \mathbb{R}$ is an eigenfunction of $G$, then the composition $f \circ p: V(H) \rightarrow \mathbb{R}$ is an eigenfunction of $H$ with the same eigenvalue. Thus, out of the $r \cdot n$ eigenvalues of $H$ (considered as a multiset), $n$ are induced from $G$ and are referred to as old eigenvalues. The other $(r-1) \cdot n$ are called the $\boldsymbol{n e w}$ eigenvalues of $H$.

Definition 1.2. Let $H$ be a covering of $G$. We say that $H$ is a Ramanujan Covering of $G$ if all the new eigenvalues of $H$ are in $[-\rho(G), \rho(G)]$. We say $H$ is a onesided Ramanujan Covering if all the new eigenvalues are bounded from above $e^{3}$ by $\rho(G)$.

\footnotetext{
${ }^{3}$ We could also define a one-sided Ramanujan covering as having all its eigenvalues bounded from below by $-\rho(G)$. Every result stated in the paper about these coverings would still hold for the lower-bound case, unless stated otherwise.
}

The existence of infinitely many $k$-regular Ramanujan graphs for every $k \geq 3$ is a long-standing open question. Bilu and Linial [2] suggest the following approach to solving this conjecture: start with your favorite $k$-regular Ramanujan graph (e.g. the complete graph on $k+1$ vertices) and construct an infinite tower of Ramanujan 2-coverings. They conjecture that every (regular) graph has a Ramanujan 2-covering. This approach turned out to be very useful in the groundbreaking result of Marcus, Spielman and Srivastava [16], who proved that every graph has a one-sided Ramanujan 2covering. This translates, as explained below, to that there are infinitely many $k$-regular bipartite Ramanujan graphs for every degree $k$.

In this paper, we generalize the result of [16] to coverings of every degree:

THEOREM 1.3. Every connected, loopless ${ }^{4}$ graph has a onesided Ramanujan $r$-covering for every $r$.

In fact, this result holds also for graphs with loops, as long as they are regular (Proposition 2.3), so the only obstruction is irregular graphs with loops. We stress that throughout this paper, all statements involving graphs hold not only for simple graphs, but also for graphs with multiple edges. Unless otherwise stated, the results also hold for graphs with loops.

A finite graph is bipartite if and only if its spectrum is symmetric around zero. In addition, every covering of a bipartite graph is bipartite. Thus, every one-sided Ramanujan covering of a bipartite graph is, in fact, a (full) Ramanujan covering. Therefore,

COROLlary 1.4. Every connected bipartite graph has a Ramanujan $r$-covering for every $r$.

In the special case where the base graph is $\rightleftharpoons$ (two vertices with $k$ edges connecting them), Theorem 1.3 and Corollary 1.4 were shown in [17], using a very different method. In this regard, our result generalizes the 2-coverings result from [16] as well as the more recent result from [17].

As we see it, the main contributions of this paper are the following:

1. This paper sheds new light on the work of MarcusSpielman-Srivastava [16]: we show there is nothing really special about $r=2$ (2-covering of graphs), and that with the right framework, the ideas can be generalized to any $r \geq 2$.

2. Our main result shows the existence of richer families of bipartite-Ramanujan graphs than was known before. For example, Corollary 1.4 implies there are $(k, \ell)$ biregular bipartite Ramanujan graphs on $(k+\ell) r$ vertices, for every $r \in \mathbb{Z}$. It also yields the existence of simple bipartite Ramanujan $k$-regular graphs of size $2 k r$ for every $r \in \mathbb{Z}$ (in contrast to [17], where the graphs are not necessarily simple). We elaborate more in Section 2.1.

3. Our method paves the way to a potentially fast construction of Ramanujan graphs. Every $r$-covering of a graph $G$ in $\mathcal{C}_{r, G}$ corresponds to a labeling of the edges of $G$ by elements of the symmetric group $S_{r}$. We generalize this notion to labeling of the edges by elements

\footnotetext{
${ }^{4}$ That is, without self-loops.
} 
of various groups and associate to every (permutation) representation of a group a family of coverings of $G$. Sometimes, this family of $r$-coverings is sparse (among all possible $r$-coverings of $G$ ). An extension of Theorem 1.3 to such cases would produce an algorithm to construct large bipartite Ramanujan graphs relatively fast. Currently, we extend Theorem 1.3 to more families of coverings (Theorem 1.11 in the full paper [10]), but not of the desired kind from this point of view. We do, however, point to some general guidelines as to which generalizations of this theorem are conceivable and which are not. See Section 4.

4. Our definition of the $r$-matching polynomial (Definition 2.5 below) and our generalization (Theorem 3.2 below) of a well-known result of Godsil and Gutman are of independent interest and can prove useful in other contexts.

This shortened version of the paper is organized as follows. In Section 2 we give some background and state a few more results. In Section 3 we give a short overview of the proof of the main result. We conclude in Section 4 with introducing a more general framework of graph coverings based on groups.

\section{BACKGROUND AND PRELIMINARY CLAIMS}

\subsection{Expander and Ramanujan Graphs}

As in Section 1, let $G$ be a finite connected graph on $n$ vertices and $A_{G}$ its adjacency matrix. Recall that $\mathfrak{p f}(G)$ is the Perron-Frobenius eigenvalue ${ }^{5}$ of $A_{G}$, that $\lambda_{n} \leq \ldots \leq \lambda_{2} \leq$ $\lambda_{1}=\mathfrak{p f}(G)$ is its spectrum, and that $\lambda(G)=\max \left(\lambda_{2},-\lambda_{n}\right)$. The graph $G$ is considered to be well-expanding if it is "highly connected". This can be measured by different combinatorial properties of $G$, most commonly by its Cheeger constant, by the rate of convergence of a random walk on $G$, and by how much the number of edges between any two sets of vertices approximates the corresponding number in a random graph (the so-called Expander Mixing Lemma) ${ }^{6}$. All these properties can be measured, at least approximately, by the spectrum of $G$, and especially by $\lambda(G)$ and the spectral gap $\mathfrak{p} f(G)-\lambda(G)$ : the smaller $\lambda(G)$ and the bigger the spectral gap is, the better expanding $G$ is ${ }^{7}$. See [12], [22, Appendix B] and the references therein.

Yet, $\lambda(G)$ cannot be arbitrarily small. The universal covering tree $T$ of $G$ is defined as follows. Choose an arbitrary vertex $v \in V(G)$. The vertices of $T$ correspond to finite non-backtracking walks in $G$ that begin in $v$. Two vertices are connected by an edge if the walk associated with one of them is a one-step extension of the walk associated with the other (see, for example, [16, Section 2.3] for a more detailed

\footnotetext{
${ }^{5}$ If $G$ is a connected graph, then the Perron-Frobenius eigenvalue $\mathfrak{p f}(G)$ of $A_{G}$ is the only eigenvalue admitting an eigenvector with strictly positive entries. In particular, $\mathfrak{p f}(G)$ is positive and $|\mu| \leq \mathfrak{p f}(G)$ for any eigenvalue $\mu$ of $A_{G}$. See, e.g., [6, Theorem 6.1].

${ }^{6}$ In this sense, well-expanding graphs resemble random graphs. The converse is also true in certain regimes of random graphs: see [22] and the references therein.

${ }^{7}$ To be precise, the Cheeger inequality relates the Cheeger constant of a graph with the value of $\lambda_{2}(G)$, and does not involve $\lambda_{n}$.
}

definition). For example, if $G$ is $k$-regular, its universal covering tree is the $k$-regular tree $\mathbb{T}_{k}$. If $T$ is the universal covering of $G$, then $G$ is called a quotient of $T$. We think of all the finite quotients of $T$ as one family of graphs. For example, for any $k \geq 2$, all finite $k$-regular graphs constitute a single such family of graphs: they are all covered by $\mathbb{T}_{k}$. It turns out that the spectral radius of $T$, denoted $\rho(G)$, plays an important role in the theory of expansion of the corresponding family of graphs. This number is the spectral radius of the adjacency operator $A_{T}$ acting on $\ell^{2}(V(T))$ by

$$
\left(A_{T} f\right)(v)=\sum_{u \sim v} f(u) .
$$

In plain terms, $\rho(G)$ can be equivalently defined as

$$
\rho(G) \stackrel{\text { def }}{=} \sup _{\substack{f \in \ell^{2}(V(T)) \\\|f\|_{2}=1}}\left\|A_{T} f\right\|_{2}
$$

where $\|f\|_{2}=\sum_{v \in V(T)} f(v)^{2}$. For the $k$-regular tree $\mathbb{T}_{k}$, this spectral radius is $2 \sqrt{k-1}$.

Theorem 2.1. [3, Theorem 6] Let $T$ be a tree with finite quotients and $\rho$ its spectral radius. For every $\varepsilon>0$, there exists $c=c(T, \varepsilon), 0<c<1$, such that if $G$ is a finite quotient of $T$ with $n$ vertices, then at least cn of its eigenvalues satisfy $\lambda_{i} \geq \rho-\varepsilon$.

In particular, $\lambda(G) \geq \rho-o_{n}$ (1) (with the $o_{n}(1)$ term depending only on $T$ ).

The last statement of the theorem, restricted to regular graphs, is due to Alon-Boppana [21]. Thus, graphs $G$ satisfying $\lambda(G) \leq \rho(G)$ are considered to be optimal expanders. Following the terminology from [15, 12], they are named Ramanujan graphs.

The seminal works $[15,18,20]$ provide an infinite family of $k$-regular Ramanujan graphs whenever $k-1$ is a prime power. Until [16] these were roughly the only known interesting families of Ramanujan graphs. Lubotzky [13, Problem 10.7.3] asked whether for every $k \geq 3$ there are infinitely many $k$-regular Ramanujan graphs ${ }^{8}$. We stress that this only hints at a much stronger phenomena. For example, it is known [5] that as $n \rightarrow \infty$, almost all $k$-regular graphs are nearly Ramanujan, in the following sense: for every $\varepsilon>0$, the non-trivial spectrum of a random $k$-regular graph falls in $[-\rho-\varepsilon, \rho+\varepsilon]$ with probability tending to 1 . Moreover, it is conjectured that among all $k$-regular graphs on $n$ vertices the proportion of Ramanujan graphs tends to a constant in $(0,1)$ as $n \rightarrow \infty$ (e.g. [19]).

Recall that we consider families of finite graphs defined by a common universal covering tree. In the regular case, every family has at least one Ramanujan graph (e.g. the complete graph on $k+1$ vertices). Other families may contain no Ramanujan graphs at all. For example, the family of $(k, \ell)$-biregular graphs, all covered by the $(k, \ell)$-biregular tree, consists entirely of bipartite graphs, so none of them is Ramanujan in the strict sense. Other families with no Ramanujan graphs, not even bipartite-Ramanujan, are shown to exist in [14]. In these cases there are certain "bad" eigenvalues outside the Ramanujan interval appearing in every finite graph in the family.

\footnotetext{
${ }^{8}$ In fact, Lubotzky's definition of Ramanujan graphs included also bipartite-Ramanujan graphs. Thus, [16] answers this question positively.
} 
Still, it makes sense to look for optimal expanders under these constraints. These are precisely those graphs where all other eigenvalues lie in the Ramanujan interval. For example, bipartite Ramanujan graphs are optimal expanders in many combinatorial senses within the set of bipartite graphs (e.g. [8, Lemma 10]). The strategy of constructing Ramanujan coverings fits this general goal: find any graph in the family which is optimal (has all its values in the Ramanujan interval except for the unavoidable bad ones) and construct Ramanujan coverings to obtain more optimal graphs in the same family. (It is easy to see that coverings of a graph $G$ are covered by the same tree as $G$.)

Marcus, Spielman and Srivastava have shown that every graph has a one-sided Ramanujan 2-covering [16]. Thus, if a family of graphs contains at least one Ramanujan graph (bipartite or not), then it has infinitely many bipartite Ramanujan graphs ${ }^{9}$. They have more recently shown that for any $k \geq 3$, the graph $\bullet \bullet$ (two vertices with $k$ edges connecting them) has a Ramanujan $r$-covering ${ }^{10}$ for every $r$ [17]. It follows there are $k$-regular bipartite Ramanujan graphs, not necessarily simple, on $2 r$ vertices for every $r$. Theorem 1.3 implies there is a richer family of bipartite Ramanujan graphs than was known before:

Corollary 2.2. Every family of graphs (defined by a common universal covering tree) containing a (simple) bipartite Ramanujan graph on $n$ vertices, also contains a (simple, respectively) bipartite Ramanujan graphs on $n r$ vertices for every $r \in \mathbb{Z}_{\geq 1}$.

In particular, there is a simple, $k$-regular, bipartite Ramanujan graph on $2 k r$ vertices for every $r$. There is also a simple, $(k, \ell)$-biregular, bipartite Ramanujan graph on $(k+\ell) r$ vertices for every $r$.

The last two statements follow by constructing Ramanujan $r$-coverings of the full $k$-regular bipartite graph on $2 k$ vertices, or of the full $(k, \ell)$-biregular bipartite graphs on $(k+\ell)$ vertices, both of which are bipartite-Ramanujan.

As of now, we cannot extend all the results in this paper to graphs with loops (and see [10, Question 6.6]). However, we can extend Theorem 1.3 to regular graphs with loops.

Proposition 2.3. Let $G$ be a regular finite graph, possibly with loops. Then $G$ has a one-sided Ramanujan $r$ covering for every $r$.

See [10, Proposition 2.3] for a full proof. We remark that in this proposition the proof does not yield the analogous result for coverings with new spectrum bounded from below by $-\rho(G)$.

\subsection{The $r$-Matching Polynomial}

An important ingredient in our proof of Theorem 1.3 is a new family of polynomials associated to a given graph. These polynomials generalize the well-known matching polynomial of a graph defined by Heilmann and Lieb [11]. Let

\footnotetext{
${ }^{9}$ Given a Ramanujan graph, its "double cover" - the 2covering with all permutations being non-identity - is bipartite Ramanujan.

${ }^{10}$ This result is stated in [17] in a different language: the authors show that there is a combination of $k$ perfect matchings between two sets of $r$ vertices, which, put together, form a $k$-regular bipartite Ramanujan graph $H$ on these $2 r$ vertices. This $H$, like every bipartite $k$-regular graph, is a covering of $\bullet \bullet$.
}

$m_{i}$ be the number of matchings in $G$ with $i$ edges, and set $m_{0}=1$. The matching polynomial of $G$ is

$$
\mathcal{M}_{G}(x) \stackrel{\text { def }}{=} \sum_{i=0}^{\lfloor n / 2\rfloor}(-1)^{i} m_{i} x^{n-2 i} \in \mathbb{Z}[x] .
$$

The following is crucially used in [16]:

Theorem 2.4. [11] $]^{11}$ The matching polynomial $\mathcal{M}_{G}$ of every finite connected graph $G$ is real-rooted with all its roots lying in the Ramanujan interval $[-\rho(G), \rho(G)]$.

Definition 2.5. Let $r \in \mathbb{Z}_{\geq 1}$. The $\mathbf{r}$-matching polynomial of a finite graph $G$, denoted $\mathcal{M}_{r, G}$, is the average of the matching polynomials of all $r$-coverings of $G$ (in $\mathcal{C}_{r, G}-$ see Definition 1.1).

For example, if $G$ is $K_{4}$ minus an edge, then

$\mathcal{M}_{3, G}(x)=x^{12}-15 x^{10}+81 x^{8}-189 x^{6}+180 x^{4}-\frac{178}{3} x^{2}+4$.

Of course, $\mathcal{M}_{1, G}=\mathcal{M}_{G}$ is the ordinary matching polynomial of $G$ (a graph is the only 1-covering of itself). Also note that these generalized matching polynomials of $G$ are monic, but their other coefficients need not be integer valued. However, they seem to share many of the nice properties of the ordinary matching polynomial. For instance,

COROLlary 2.6. All real roots of $\mathcal{M}_{r, G}$ are inside the $R a$ manujan interval $[-\rho(G), \rho(G)]$.

Proof. Recall that $n$ denotes the number of vertices of $G$. The ordinary matching polynomial of every $H \in \mathcal{C}_{r, G}$ is a degree- $n r$ monic polynomial. As every $r$-covering of $G$ is covered by the same universal covering tree as $G$, we obtain by Theorem 2.4 that $\mathcal{M}_{H}$ is strictly positive in the interval $(\rho(G), \infty)$, and is either strictly positive or strictly negative in $(-\infty,-\rho(G))$ depending only on the parity of $n r$. The corollary now follows by the definition of $\mathcal{M}_{r, G}$ as the average of such polynomials.

In fact, Theorem 2.4 can be generalized in full to $\mathcal{M}_{r, G}$ for every $r$ :

THEOREM 2.7. Let $G$ be a finite, connected, loopless graph. For every $r \in \mathbb{Z}_{\geq 1}$, the polynomial $\mathcal{M}_{r, G}$ is real rooted with all its roots contained in the Ramanujan interval $[-\rho(G), \rho(G)]$.

Theorem 2.7 would follow from Corollary 2.6 by showing that $\mathcal{M}_{r, G}$ is real rooted. We do not have a direct proof of this fact: the proof we present here is a byproduct of the proof we present for Theorem 1.3 - see Section 3 .

Another ingredient in the proof of Theorems 1.3 and 2.7 is a precise formula for $\mathcal{M}_{r, G}$ as a weighted generating function for " $r$-multi-matchings" of $G$. An $r$-multi-matching of a graph $G$ is a multiset of edges which covers every vertex at most $r$ times. Formally, a multi-matching is a function $m: E(G) \rightarrow \mathbb{Z}_{\geq 0}$ with $m(-e)=m(e)$ for every $e \in E(G)$. We denote by $e_{v, 1}, \ldots, e_{v, \operatorname{deg}(v)}$ the edges in $E(G)$ emanating from a vertex $v \in V(G)$ (in an arbitrary order, loops at

\footnotetext{
${ }^{11}$ Actually, [11] shows that $\mathcal{M}_{G}$ satisfies this statement only when $G$ is regular. Apparently, the case of irregular graphs was first noticed in [16], even though some of the original proofs of [11] work in the irregular case just as well.
} 
$v$ appearing twice, of course), and by $m(v)$ the number of edges incident with $v$ in the multi-matching. Namely,

$$
m(v)=\sum_{i=1}^{\operatorname{deg}(v)} m\left(e_{v, i}\right) .
$$

Finally, we denote by $|m|$ the total number of edges in $m$ (with multiplicity), so $|m|=\sum_{e \in E^{+}(G)} m(e)$. We say that $m$ is an $r$-multi-matching if $m(v) \leq r$ for every $v \in V(G)$.

Proposition 2.8. For every $r$-multi-matching $m$ of $G$ denote $^{12}$

$$
W_{r}(m)=\frac{\prod_{v \in V(G)}\left(\begin{array}{c}
r \\
m\left(e_{v, 1}\right), \ldots, m\left(e_{v, \operatorname{deg}(v)}\right)
\end{array}\right)}{\prod_{e \in E^{+}(G)}\left(\begin{array}{c}
r \\
m(e)
\end{array}\right)} .
$$

Then,

$$
\mathcal{M}_{r, G}(x)=\sum_{m: \text { an } r-\text { multi-matching }}(-1)^{|m|} \cdot W_{r}(m) \cdot x^{n r-2|m|} .
$$

The proof of this formula for $\mathcal{M}_{r, G}$ can be found in [10, Section 2.2].

\section{OVERVIEW OF THE PROOF}

We now give an overview of the proof of Theorem 1.3. For simplicity, we assume that $G$ is a simple graph (namely, does not have multiple edges). We also focus on the case $r=3$, although we try to give the statements for general $r$ whenever this does not reduce readability. Our goal, therefore, is to prove that every connected simple graph $G$ admits a one-sided Ramanujan 3-covering.

\subsection{Ingredient I: Isolate the New Spectrum}

Let $G$ be a connected graph on $n$ vertices and let $H \in \mathcal{C}_{3, G}$ be a 3 -covering of $G$ associated to $\sigma: E(G) \rightarrow S_{3}$. The $3 n \times 3 n$ adjacency matrix $A_{H}$ of $H$ can be obtained as a "blow-up" of $A_{G}$, where every entry is blown up to a $3 \times 3$ block. We write $\sigma(e)$ as a $3 \times 3$ permutation matrix instead of the entry of $A_{G}$ corresponding to $e \in E(G)$. A zero entry in $A_{G}$ gets blown up to a zero $3 \times 3$ block.

The first step of the proof consists of changing the basis of $A_{H}$. Let $\delta_{v}$ denote the unit elementary vector with 1 at the $v$ coordinate. Instead of using the basis $\bigcup_{v \in V(G)}\left\{\delta_{v_{1}}, \delta_{v_{2}}, \delta_{v_{3}}\right\}$, we use the basis

$$
\begin{aligned}
& {\left[\bigcup_{v \in V(G)}\left\{\delta_{v_{1}}+\omega \delta_{v_{2}}+\omega^{2} \delta_{v_{3}}, \delta_{v_{1}}+\omega^{2} \delta_{v_{2}}+\omega \delta_{v_{3}}\right\}\right] \cup } \\
& {\left[\bigcup_{v \in V(G)}\left\{\delta_{v_{1}}+\delta_{v_{2}}+\delta_{v_{3}}\right\}\right] }
\end{aligned}
$$

where $\omega=e^{\frac{2 \pi i}{3}}$ is a primitive third root of unity. With this new basis, $A_{H}$ becomes a block-diagonal matrix, with one block of size $2 n \times 2 n$ and the other one of size $n \times n$ and identical to $A_{G}$. We illustrate this is Figure 2.

With this change of basis, the $n$ "old" eigenvalues of $H$, namely, those inherited from $G$, are conveniently found in ${ }^{12}$ We use the notation $\left(\begin{array}{c}b \\ a_{1}, a_{2}, \ldots, a_{k}\end{array}\right)$ to denote the multinomial coefficient $\frac{b !}{a_{1} ! \ldots a_{k} !\left(b-\sum a_{i}\right) !}$.

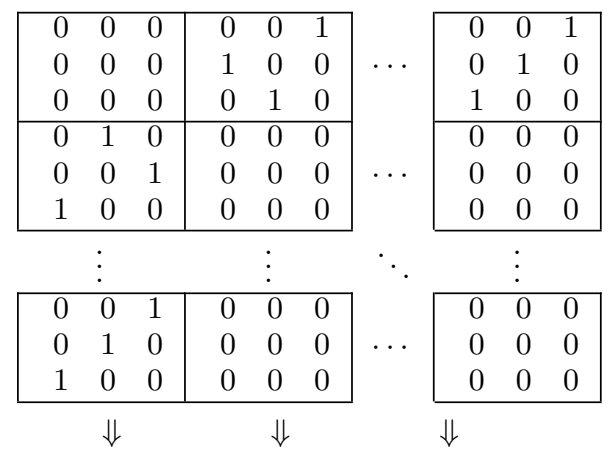

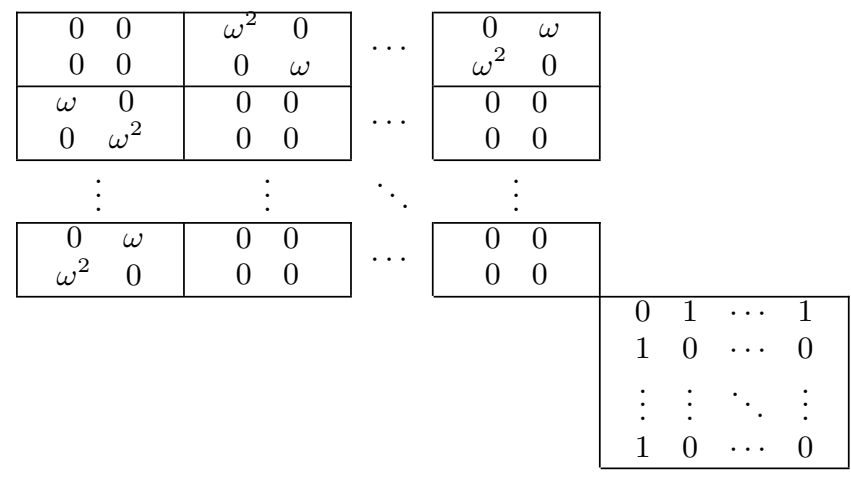

Figure 2: A change of basis of $A_{H}$

the $n \times n$ block at the bottom, while the $2 n$ "new" eigenvalues are the eigenvalues of the top $2 n \times 2 n$ block. The $2 \times 2$ block associated with the permutation $\sigma(e) \in S_{3}$ is the image of $\sigma(e)$ under the "standard" representation of $S_{3}$, denoted std, which is a homomorphism of groups std: $S_{3} \rightarrow \mathrm{GL}_{2}(\mathbb{C})$. (See [10, Section 2.4] for more details.) For this reason, we name the $2 n \times 2 n$ block $A_{\sigma, \text { std }}$. Our goal then is to show there is some 3-covering of $G$, namely, some $\sigma: E(G) \rightarrow S_{3}$ (with $\sigma(-e)=\sigma(e)^{-1}$, of course), such that all eigenvalues of $A_{\sigma, \text { std }}$ are bounded from above by $\rho(G)$.

For general $r$, this step consists of a change of basis of $A_{H}$ which makes it a block-diagonal matrix with an $(r-1) n \times$ $(r-1) n$ block plus an $n \times n$ block identical to $A_{G}$. The $(r-1) n \times(r-1) n$ block corresponds to the standard representation of $S_{r}$. In the case $r=2$, the standard representation of $S_{2}$ coincides with the sign representations, and the analogous decomposition of $A_{H}$ in this case is used already in [2] and in [16].

\subsection{Ingredient II: Convex Combinations of $r$ - Coverings}

The starting point of the strategy of [16] is that instead of considering "discrete" coverings of the graph $G$, one can consider convex combinations of coverings, or more precisely, convex combinations of characteristic polynomials of coverings. Concretely, let $\Delta_{r}(G)$ be the simplex of all probability distributions on $r$-coverings of the graph $G$. The simplex $\Delta_{r}(G)$ has $\left|\mathcal{C}_{r, G}\right|=(r !)\left|E^{+}(G)\right|$ vertices. To the vertex 
corresponding to the $r$-covering $H \in \mathcal{C}_{r, G}$ we associate the characteristic polynomial of the new spectrum of $H$, namely,

$$
\begin{aligned}
\phi_{H} & =\frac{\operatorname{det}\left(x I-A_{H}\right)}{\operatorname{det}\left(x I-A_{G}\right)}=\prod_{\mu \in \text { New Spectrum of } H}(x-\mu) \\
& =\operatorname{det}\left(x I-A_{\sigma, \mathrm{std}}\right)
\end{aligned}
$$

where $\sigma: E(G) \rightarrow S_{r}$ is the labeling of $G$ corresponding to $H$. Every point $p \in \Delta_{r}(G)$ is associated with a polynomial $\phi_{p}$, the corresponding weighted average of the $\left\{\phi_{H}\right\}_{H \in \mathcal{C}_{r, G}}$ : if $p=\sum_{H \in \mathcal{C}_{r, G}} \alpha_{H} \cdot H$, then $\phi_{p}=\sum_{H \in \mathcal{C}_{r, G}} \alpha_{H} \cdot \phi_{H}$. Our goal is to find a vertex $v$ of $\Delta_{r}(G)$ with all roots of $\phi_{v}$ bounded from above by $\rho$. To obtain this we look for more points in the simplex with this property.

\subsection{Ingredient III: Find a "Ramanujan Point" in $\Delta_{r}(G)$}

We say that a point $p$ in the simplex $\Delta_{r}(G)$ is Ramanujan if the associated polynomial $\phi_{p}$ is real rooted with all its roots lying inside the Ramanujan interval $[-\rho, \rho]$. As we explain below, in order to find a one-sided Ramanujan vertex, the strategy of [16] requires to first find some one-sided Ramanujan point in the simplex.

In the $r=2$ case, the center point of $\Delta_{2}(G)$, corresponding to the uniform distribution on 2-coverings, is Ramanujan. This is based on [7], who observed that the corresponding polynomial is equal to the matching polynomial $\mathcal{M}_{G}$, and on Theorem 2.4 above, due to [11], by which $\mathcal{M}_{G}$ is real rooted with all its roots inside $[-\rho, \rho]$. We generalize this to every $r$ :

THEOREM 3.1. The center point of $\Delta_{r}(G)$ is Ramanujan for every $r \geq 2$.

The proof of Theorem 3.1 consists of two main parts. The first one is a generalization of the observation from [7]:

TheOREM 3.2. The polynomial corresponding to the center point of $\Delta_{r}(G)$ is the $(r-1)$-matching polynomial $\mathcal{M}_{r-1, G}$

$$
\mathbb{E}_{\sigma \sim \mathcal{C}_{r, G}}\left[\operatorname{det}\left(x I-A_{\sigma, \mathrm{std}}\right)\right]=\mathcal{M}_{r-1, G}(x) .
$$

Consider the $(r-1) \times(r-1)$ blocks in the random matrix $x I-A_{\sigma, \text { std }}$ with $\sigma \sim \mathcal{C}_{r, G}$. The only dependencies are between the $(i, j)$-block and the $(j, i)$-block, corresponding to an edge $e$ and its inverse $-e$. Otherwise, the blocks are independent.

In the $r=2$ case, these are $1 \times 1$ blocks, each either deterministically zero or a \pm 1 entry with zero expectation. The argument in [7] proceeds as follows: if one expands the left hand side of (3.2) as a sum of generalized diagonals, then every diagonal containing the $(i, j)$-entry but not the $(j, i)$-entry is zero in expectation. Thus, the non-vanishing generalized diagonals are precisely those corresponding to matchings in $G$. This simple argument is written in full also in [16, Appendix A].

When $r \geq 3$ the result is new and the proof more involved. The main ideas in the proof are the following:

1. We also expand the left hand side of (3.2) as a sum over generalized diagonals, but then, for a given generalized diagonal $\pi \in S_{(r-1) n}$, we consider the entries it "uses" from every $(r-1) \times(r-1)$ block and the smallest minor (square sub-matrix) of the block these entries are contained in. When $r=3$, there are three possibilities for a given $2 \times 2$ block $B$ :

- either $\pi$ contains no entries from $B$, and then the associated minor is the empty one, or

- $\pi$ contains one entry from $B$, in which case the associated minor is a $1 \times 1$ minor consisting of this very entry, or

- $\pi$ contains two entries from $B$, and then the associated minor is the entire $2 \times 2$ block.

We then group together all generalized diagonals with the same array of associated minors. Using this, we find a way to write $\mathbb{E}_{\sigma \sim \mathcal{C}_{r, G}}\left[\operatorname{det}\left(x I-A_{\sigma, \mathrm{std}}\right)\right]$ as an expression consisting of determinants of minors of blocks - see [10, Lemma 3.1].

2. Using the independence of small blocks (except for the $(i, j)$-block and the $(j, i)$-block), we reduce to an expression involving sum and products of terms of the form

$\mathbb{E}\left[\operatorname{det}\left(\right.\right.$ some minor of $\left.B_{i, j}\right) \cdot \operatorname{det}\left(\right.$ some minor of $\left.\left.B_{j, i}\right)\right]$

where $B_{i, j}$ is the $(i, j)$-block of size $(r-1) \times(r-1)$ in $\left(x I-A_{\sigma, \mathrm{std}}\right)-$ see [10, Equation (3.4)].

3. We apply the classical Peter-Weyl theorem from the theory of group representations, as well as some wellknown facts about the representations of the symmetric group. In our context, those can be interpreted as saying that (3.3) is zero unless the minor of $B_{i, j}$ is the transpose of the minor of $B_{j, i}$. It also gives the precise expectation in the case one minor is the transpose of the other.

We illustrate this when $r=3$. The value of std for every permutation $\tau \in S_{3}$ is listed in the second column of Table 1. Notice that $\operatorname{std}\left(\tau^{-1}\right)$ is always the conjugate-transpose of $\operatorname{std}(\tau)$ (this is not a coincidence - with the basis we chose in (3.1), the image of std lies in the unitary subgroup $\left.U(2) \leq \mathrm{GL}_{2}(\mathbb{C})\right)$. If the edge $e=(i, j)$ in $G$ is labeled by $\tau$, then $-e=(j, i)$ is labeled by $\tau^{-1}$, and so $B_{j, i}$ is always the conjugatetranspose of $B_{i, j}$. The claim about the values of (3.3) is therefore equivalent to that

$\mathbb{E}\left[\operatorname{det}\left(\right.\right.$ some minor of $\left.\left.B_{i, j}\right) \cdot \overline{\operatorname{det}\left(\text { some minor of } B_{i, j}\right)}\right]$

is non-zero if and only if the two minors are the same. This can be easily verified for the case $r=3$ using Table 1: there are six possible minors, and for every pair of two different ones (3.4) vanishes. For instance, if the first minor is the top-left $1 \times 1$ minor and the second is the bottom-right $1 \times 1$ one, (3.4) becomes

$$
\frac{1 \cdot \overline{1}+\omega^{2} \cdot \bar{\omega}+\omega \cdot \overline{\omega^{2}}+0 \cdot \overline{0}+0 \cdot \overline{0}+0 \cdot \overline{0}}{6}=0 .
$$

Note that if the two minors are the same, the expectation is not zero: it is $\frac{1}{2}$ for every $1 \times 1$ minor, and 1 for the empty minor as well as for the $2 \times 2$ minor.

4. Finally, we show the resulting expression is equal to the formula (2.2) we have for $\mathcal{M}_{r, G}$. 
Table 1: The standard representation of $S_{3}$ and the determinants of its minors

\begin{tabular}{|c||c|c|c|}
\hline$\tau \in S_{3}$ & $\operatorname{std}(\tau)$ & $\begin{array}{c}\text { determinant } \\
\text { of empty } \\
\text { minor }\end{array}$ & $\begin{array}{c}\text { determinant } \\
\text { of } 2 \times 2 \text { minor }\end{array}$ \\
\hline \hline id & $\left(\begin{array}{cc}1 & 0 \\
0 & 1\end{array}\right)$ & 1 & 1 \\
\hline$(123)$ & $\left(\begin{array}{cc}\omega^{2} & 0 \\
0 & \omega\end{array}\right)$ & 1 & 1 \\
\hline$(132)$ & $\left(\begin{array}{cc}\omega & 0 \\
0 & \omega^{2}\end{array}\right)$ & 1 & -1 \\
\hline$(12)$ & $\left(\begin{array}{cc}0 & \omega^{2} \\
\omega & 0\end{array}\right)$ & 1 & -1 \\
\hline$(13)$ & $\left(\begin{array}{cc}0 & \omega \\
\omega^{2} & 0\end{array}\right)$ & 1 & -1 \\
\hline$(23)$ & $\left(\begin{array}{cc}0 & 1 \\
1 & 0\end{array}\right)$ & 1 & 1 \\
\hline
\end{tabular}

The full proof of Theorem 3.2 is given in Section 3 of the full paper [10].

Recall that our intermediate goal is to show Theorem 3.1, namely, that the center point of $\Delta_{r}(G)$ is Ramanujan. In the case $r=2$, Theorems 3.2 and 2.4 immediately yield this. When $r \geq 3$, they only yield that all the real roots of the polynomial associated with the center point are in $[-\rho, \rho]$ (see Corollary 2.6). We still need to prove this polynomial has only real roots. This is established by showing that the center point belongs to a real-rooted zone in $\Delta_{r}(G)$, which we explain below.

\subsection{Ingredient IV: Real-Rooted Zone in $\Delta_{r}(G)$}

The following theorem shows that for certain distributions of $r$-coverings of $G$, the corresponding average characteristic polynomial in $\Delta_{r}(G)$ is real rooted. It is a slight variation on results from $[17$, Section III]:

THEOREM 3.3. Let $p \in \Delta_{r}(G)$ be a distribution over the set of $r$-coverings of $G$, and suppose that:

1. The random permutation-valued variables $\{\sigma(e)\}_{e \in E^{+}(G)}$ associated with the positively oriented edges $E^{+}(G)$ are completely independent of each other, and

2. For every $e \in E^{+}(G)$, the random variable $\sigma(e)$ equals a product of independent variables $X_{e, 1} X_{e, 2} \ldots X_{e, r(e)}$, each $X_{e, i}$ having (at most) two values: the identity, and some transposition ${ }^{13}$.

Then $\phi_{p}=\mathbb{E}_{\sigma \sim p}\left[\operatorname{det}\left(x I-A_{\sigma, \mathrm{std}}\right)\right]$ is real rooted

When $r=2$, every distribution on 2-coverings trivially satisfies the second property: $\sigma(e)$ is always some distribution over $S_{2}=\{\mathrm{Id},(12)\}$. So the $r=2$ analogue of this theorem is slightly simpler, and is the content of Theorem 5.1 in [16]. To see that the second property is crucial when $r \geq 3$, consider, for instance, the cycle of length two $\longrightarrow$. Define a distribution $p$ on its 3-coverings by labeling one edge deterministically with the identity permutation and the other edge with either the identity or a 3-cycle (123), each

\footnotetext{
${ }^{13}$ A transposition in $S_{r}$ is a permutation which swaps two
} elements in $\{1, \ldots, r\}$ and leaves all other ones fixed. with probability $\frac{1}{2}$. The average characteristic polynomial of $A_{\sigma, \text { std }}$ is then

$$
\frac{\left(x^{2}-4\right)^{2}+\left(x^{2}-1\right)^{2}}{2}
$$

which is not real rooted. A proof of a more general version of Theorem 3.3 appears in Section 4 of the full paper (Proposition 4.4).

There are two crucial properties of the real-rooted zone of $\Delta_{r}(G)$ we get from Theorem 3.3: first, it contains the center point which is thus a Ramanujan point (see Ingredient $\mathrm{V}$ below), and it contains straight intervals which we use in Ingredient VI.

\subsection{Ingredient V: The Real-Rooted Zone Con- tains the Center Point of $\Delta_{r}(G)$}

The following clever observation basically appears in [17]:

Proposition 3.4. The uniform distribution over all $r$ coverings of $G$ satisfies the assumptions of Theorem 3.3. It follows that the center point of $\Delta_{r}(G)$ is real rooted.

Hence by Theorem 3.2 and Corollary 2.6, the center point is Ramanujan. This proves Theorems 3.1 and 2.7. Note that Proposition 3.4 holds trivially when $r=2$, and this yields yet another proof for the real-rootedness of the matching polynomial $\mathcal{M}_{G}$.

In the full paper, since we give a more general result about coverings based on group representations (see Section 4 here), our proof of Proposition 3.4 is not constructive. However, for the special case of the family of all $r$-coverings and the symmetric group $S_{r}$, a constructive proof to this fact is given in [17, Lemma III.5]. For example, when $r=3$, define

$$
\begin{aligned}
& X= \begin{cases}\mathrm{Id} & \text { with prob } \frac{1}{2} \\
(12) & \text { with prob } \frac{1}{2}\end{cases} \\
& Y= \begin{cases}\mathrm{Id} & \text { with prob } \frac{1}{3} \\
(13) & \text { with prob } \frac{2}{3}\end{cases} \\
& Z= \begin{cases}\mathrm{Id} & \text { with prob } \frac{1}{2} \\
(12) & \text { with prob } \frac{1}{2}\end{cases}
\end{aligned}
$$

Then, the random permutation $X \cdot Y \cdot Z$ has the uniform distribution in $S_{3}$ while satisfying the second property in Theorem 3.3. For general $r$, the construction in [17, Lemma III.5] consists of a product of $2^{r-1}-1$ random variables. This can be reduced to a product of $\left(\begin{array}{l}r \\ 2\end{array}\right)$ random variables see [10, Remark 4.7].

\subsection{Ingredient VI: A Tree of Interlacing Poly- nomials}

We reach the endgame. A key point in the strategy of [16] is the following elementary yet very useful fact:

FaCt 3.5 (E.G. Proof of [16, Lemma 4.2]). Assume that $f, g \in \mathbb{R}[x]$ are two polynomials of degree $n$ so that $(1-\lambda) f+\lambda g$ is real rooted for every $\lambda \in[0,1]$. Then, for every $1 \leq i \leq n$, the $i$-th root of $(1-\lambda) f+\lambda g$ moves monotonically as $\lambda$ moves from 0 to 1 .

Namely, if the roots of a polynomial $h$ are all real and denoted $r_{n}(h) \leq \ldots \leq r_{2}(h) \leq r_{1}(h)$, then Fact 3.5 means 


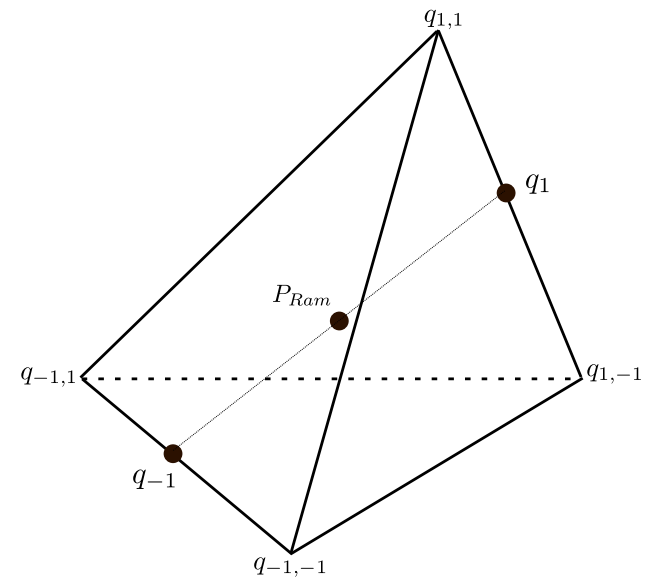

Figure 3: The simplex $\Delta_{2}(G)$ when $\left|E^{+}(G)\right|=2$

that the function $\lambda \mapsto r_{i}((1-\lambda) f+\lambda g)$ is monotone (nondecreasing or non-increasing) for every $i$. The assumption in Fact 3.5 is equivalent to that $f$ and $g$ have a "common interlacing". We elaborate in [10, Section 2.5].

The crux of the matter is that among the family of distributions in the real-rooted zone of $\Delta_{r}(G)$ described in Theorem 3.3, if one perturbs the distribution of only one of the variables $X_{e, i}$, the corresponding point in $\Delta_{r}(G)$ moves along a straight interval, which allows us to use Fact 3.5.

We illustrate this in the case $r=2$, where the distribution of every edge is determined by a single random variable $X_{e}$. Order the edges in $E^{+}(G)$ by $e_{1}, e_{2}, \ldots$ in an arbitrary fashion. Start at the Ramanujan point $p_{\text {Ram }}$ - the center point of $\Delta_{2}(G)$. Let $q_{1}$ denote the point in $\Delta_{2}(G)$ where $e_{1}$ is labeled by id $\in S_{2}$ and all remaining edges are labeled uniformly at random and independently. Let $q_{-1}$ be another point with the same definition except that $e_{1}$ is labeled by $(12) \in S_{2}$. Now $p_{R a m}$ lies on the straight interval connecting $q_{1}$ and $q_{-1}$. Note that every point on this interval corresponds to a random 2-covering in which every edge is labeled independently from the others, and the associated polynomial is, therefore, real rooted. By Fact 3.5, the largest root of $\phi_{p_{R a m}}$ lies between the largest root of $\phi_{q_{1}}$ and the largest root of $\phi_{q_{-1}}$. Hence, one of the two points $q_{1}$ or $q_{-1}$ has largest root at most the one of $p_{R a m}$, and in particular at most $\rho$. Assume without lost of generality that $q_{-1}$ has largest root at most $\rho$. Now repeat this process, this time by perturbing the distribution on $e_{2}$. Let $q_{-1,1} \in \Delta_{2}(G)$ denote the point where $\sigma\left(e_{1}\right)=(12), \sigma\left(e_{2}\right)=$ id and all remaining edges are distributed uniformly and independently, and let $q_{-1,-1}$ be defined similarly, only with $\sigma\left(e_{2}\right)=(12)$. Since $q_{-1}$ lies on the straight line between $q_{-1,1}$ and $q_{-1,-1}$, the largest root of one of these two latter points is at most that of $q_{-1}$. If we go on and gradually choose a deterministic value for every $e \in E^{+}(G)$ while not increasing the largest root, we end up with a vertex of $\Delta_{2}(G)$ representing a one-side Ramanujan 2-covering of $G$. This is illustrated in Figure 3.

To find a one-sided Ramanujan $r$-covering of $G$ in the general case (for arbitrary $r$ ), we imitate the process illustrated in Figure 3, only at each stage we perturb and then fix the value of one of the independent random variables $X_{e, i}$. For example, in the case $r=3$ and the variables constructed in (3.5), this translates to the $3\left|E^{+}(G)\right|$-step process illus-
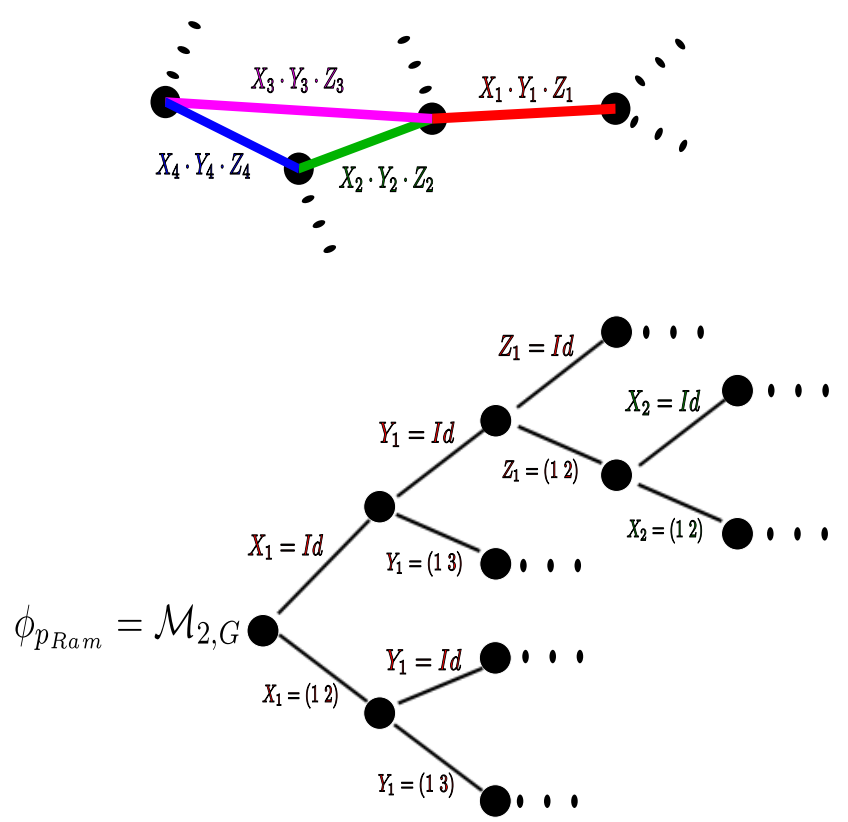

Figure 4: On the top is a piece of the graph $G$ with three random variables associated to every edge. On the bottom is a piece of the corresponding binary tree of interlacing polynomials.

trated in Figure 4. At each step, we determine the value of one of the $3\left|E^{+}(G)\right|$ variables so that the maximal root of the associated polynomial never increases.

\section{GENERALIZING TO OTHER GROUP REPRESENTATIONS}

As explained above, every $r$-covering of $G$ corresponds to some labeling of the edges of $G$ by permutations from $S_{r}$. We study, more generally, the following problem:

Question 4.1. Let $\Gamma \leq S_{r}$ be a subgroup. Does every connected graph $G$ have a (one-sided / full) Ramanujan $r$ covering $\sigma: E(G) \rightarrow S_{r}$ with all permutations belonging to $\Gamma$ (i.e. with $\sigma(E(G)) \subseteq \Gamma)$ ?:

Even more generally, we ask a similar question for any finite group $\Gamma$ together with any representation, namely, any group homomorphism $\pi: \Gamma \rightarrow \mathrm{GL}_{d}(\mathbb{C})$ [10, Question 1.6]. We point to two group-theoretic properties of $\Gamma$ and $\pi$ which are required for the proof described in Section 3 to work [10, Section 1.2].

We also point to some limitations. For example, in $[10$, Remark 1.14] we explain why one cannot hope to have Ramanujan coverings when $\Gamma$ is a cyclic group (a.k.a. "shiftlifts") or more generally when $\Gamma$ is abelian (unless it is of very small order).

In contrast, we do not know what the answer may be for the following compelling example. Let $q$ be a prime power and $\mathbb{F}_{q}$ the field with $q$ elements. Consider the group $\mathrm{PSL}_{2}\left(\mathbb{F}_{q}\right)$, which consists of all $2 \times 2$ matrices $A$ with entries from $\mathbb{F}_{q}$ and $\operatorname{det}(A)=1$ where $A$ and $-A$ are identified. This group acts by permutations on the projective line $\mathbb{P}^{1}\left(\mathbb{F}_{q}\right)$, which consists of all 1-dimensional subspaces in $\mathbb{F}_{q}^{2}$ 
and is of size $q+1$ : for $v \in \mathbb{F}_{q}^{2} \backslash\{0\}$ and $A \in \operatorname{PSL}_{2}\left(\mathbb{F}_{q}\right)$, the permutation defined by $A$ maps the line $\left\{\lambda v \mid \lambda \in \mathbb{F}_{q}\right\}$ to the line $\left\{\lambda A v \mid \lambda \in \mathbb{F}_{q}\right\}$. This gives an embedding of $\mathrm{PSL}_{2}\left(\mathbb{F}_{q}\right)$ as a subgroup of $S_{q+1}$.

Question 4.2. Let $q$ be a prime power. Does every connected graph $G$ have a (one-sided / full) Ramanujan $(q+1)$ covering $\sigma: E(G) \rightarrow S_{q+1}$ with all permutations belonging to $\mathrm{PSL}_{2}\left(\mathbb{F}_{q}\right)$ ?

A positive answer to this question (for every $q$ ) would yield a fast construction of large Ramanujan graphs: there are only around $q^{3}$ elements in $\mathrm{PSL}_{2}\left(\mathbb{F}_{q}\right)$. Thus, the number of $(q+1)$-coverings of $G$ in the family defined by this subgroup of $S_{q+1}$ is around $q^{3|E(G)|}$. If we want $k$-regular bipartite-Ramanujan graphs of some constant degree $k$, we could construct them in polynomial time in the number of vertices. For example, we could find a Ramanujan $(q+1)$ covering of $\bullet$ in time, roughly, $q^{3 k}$. We find this example especially compelling because this subgroup of permutations is sparse and well-understood, and also because the group $\operatorname{PSL}_{2}(q)$ has proven useful before in constructing Ramanujan graphs: the explicit construction of Ramanujan graphs in $[15,18]$ uses Cayley graphs of such groups. Unfortunately, the group $\mathrm{PSL}_{2}\left(\mathbb{F}_{q}\right)$ and its representation described above do not satisfy the group-theoretic properties our proof requires, so the results in this paper do not apply. So far, we have not conducted significant simulations in this direction.

For more details about this line of research, see [10], especially Sections 1.2 and 5 and Questions 6.1-6.4 in Section 6.

\section{ACKNOWLEDGMENTS}

We thank the anonymous referees for valuable comments.

\section{REFERENCES}

[1] A. Amit and N. Linial. Random graph coverings I: General theory and graph connectivity. Combinatorica, 22(1):1-18, 2002.

[2] Y. Bilu and N. Linial. Lifts, discrepancy and nearly optimal spectral gap. Combinatorica, 26(5):495-519, 2006.

[3] S. M. Cioabă. Eigenvalues of graphs and a simple proof of a theorem of Greenberg. Linear algebra and its applications, 416(2):776-782, 2006.

[4] J. Friedman. Relative expanders or weakly relatively Ramanujan graphs. Duke Mathematical Journal, 118(1):19-35, 2003.

[5] J. Friedman. A proof of Alon's second eigenvalue conjecture and related problems. Mem. Amer. Math. Soc., 195(910):viii+100, 2008.

[6] C. D. Godsil. Algebraic combinatorics. CRC Press, 1993.
[7] C. D. Godsil and I. Gutman. On the matching polynomial of a graph. In L. Lovász and V. Sós, editors, Algebraic Methods in Graph Theory, volume 25 of Colloquia Mathematica Societatis János Bolyai, pages 241-249. János Bolyai Mathematical Society, 1981.

[8] K. Golubev and O. Parzanchevski. Spectrum and combinatorics of Ramanujan triangle complexes. arXiv preprint arXiv:1406.6666, 27 pages, 2014.

[9] Y. Greenberg. On the spectrum of graphs and their universal coverings, (in Hebrew). PhD thesis, Hebrew University, 1995.

[10] C. Hall, D. Puder, and W. F. Sawin. Ramanujan coverings of graphs. arXiv preprint arXiv:1506.02335v2, 2015.

[11] O. J. Heilmann and E. H. Lieb. Theory of monomer-dimer systems. Communications in Mathematical Physics, 25(3):190-232, 1972.

[12] S. Hoory, N. Linial, and A. Wigderson. Expander graphs and their applications. Bulletin of the American Mathematical Society, 43(4):439-562, 2006.

[13] A. Lubotzky. Discrete Groups, Expanding Graphs and Invariant Measures, volume 125 of Progress in Mathematics. Birkhauser, 1994.

[14] A. Lubotzky and T. Nagnibeda. Not every uniform tree covers Ramanujan graphs. Journal of Combinatorial Theory, Series B, 74(2):202-212, 1998.

[15] A. Lubotzky, R. Phillips, and P. Sarnak. Ramanujan graphs. Combinatorica, 8(3):261-277, 1988.

[16] A. Marcus, D. A. Spielman, and N. Srivastava. Interlacing families I: Bipartite Ramanujan graphs of all degrees. Annals of Mathematics, 182(1):307-325, 2015. appeared also in FOCS 2013.

[17] A. Marcus, D. A. Spielman, and N. Srivastava. Interlacing families IV: Bipartite Ramanujan graphs of all sizes. In Foundations of Computer Science (FOCS), 2015 IEEE 56th Annual Symposium on, pages 1358-1377. IEEE, 2015. arXiv version:1505.08010.

[18] G. A. Margulis. Explicit group-theoretic constructions of combinatorial schemes and their applications in the construction of expanders and concentrators. Problemy Peredachi Informatsii, 24(1):51-60, 1988.

[19] S. J. Miller, T. Novikoff, and A. Sabelli. The distribution of the largest nontrivial eigenvalues in families of random regular graphs. Experimental Mathematics, 17(2):231-244, 2008.

[20] M. Morgenstern. Existence and explicit constructions of $\mathrm{q}+1$ regular Ramanujan graphs for every prime power q. Journal of Combinatorial Theory, Series B, 62(1):44-62, 1994.

[21] A. Nilli. On the second eigenvalue of a graph. Discrete Mathematics, 91(2):207-210, 1991.

[22] D. Puder. Expansion of random graphs: New proofs, new results. Inventiones Mathematicae, 201(3):845-908, 2015. 